\title{
A Comparison of Different Options to Improve PDM-QPSK Resilience against Cross-channel Nonlinearities
}

\author{
Donato Sperti, Paolo Serena and Alberto Bononi \\ Università degli Studi di Parma, Dipartimento di Ingegneria dell'Informazione, viale G. Usberti 181/A, \\ 43124 Parma (Italy), 凶 donato.sperti@nemo.unipr.it
}

\begin{abstract}
Q-factor improvements induced by channel walk-off coming from either chromatic dispersion, or PMD, or in-line XPM suppressors are compared in $112 \mathrm{~Gb} / \mathrm{s} P D M-Q P S K$ WDM transmissions with $N R Z$, aligned-RZ and interleaved-RZ pulses.
\end{abstract}

\section{Introduction}

Polarization division multiplexing (PDM) - quadrature phase shift keying (QPSK) has emerged as one of the most attractive solutions for 100 $\mathrm{Gb} / \mathrm{s}$ transmissions. While single-channel linear impairments can be almost completely compensated by means of digital signal processing (DSP) based coherent detection, the performance of wavelength division multiplexing (WDM) transmissions on a $50 \mathrm{GHz}$ grid is set by crosschannel fiber nonlinearities ${ }^{1}$. As a way to mitigate cross-channel effects, some experiments ${ }^{2}$ and simulations ${ }^{1}$ verified the benefits of the interleaved return-to-zero (iRZ) pulse format, in which the polarization tributaries are $50 \%-R Z$ shaped and delayed by half a symbol time. Polarization mode dispersion (PMD) should reduce the iRZ benefits by re-aligning the polarizations. However, PMD also introduces channel depolarization, thus reducing cross-channel effects, when linear PMD is fully compensated at the receiver ${ }^{3}$. Hence a quantitative analysis of the PMD impact on $\mathrm{iRZ}$ transmission is of great interest. Crosschannel nonlinearities can also be mitigated by the fiber group velocity dispersion (GVD), which, especially in links without dispersion management (noDM), induces substantial channel walkoff $^{1,4}$. Another efficient way to increase channel walk-off is to use passive devices that introduce different delays on adjacent channels at specific points of the line: such devices were introduced to efficiently suppress cross-phase modulation (XPM) in on-off keying (OOK) systems ${ }^{5}$. XPM suppressors based on periodic group-delay have already been successfully tested for PDM-QPSK systems ${ }^{6}$, thus proving that they are effective not only against XPM, but also against another fundamental impairment of PDM systems, namely, cross-polarization modulation $(\mathrm{XPolM})^{7}$.

In this paper we compare the effectiveness of PMD, of GVD, and of the XPM suppressor in

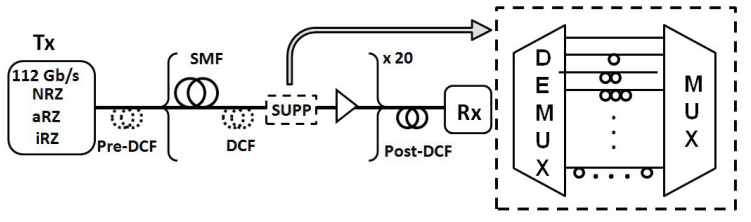

Fig. 1: System simulation setup.

mitigating cross-channel nonlinearities in PDMQPSK transmissions for three different pulse formats: iRZ, non-return to zero (NRZ) and aligned $\mathrm{RZ}$ (aRZ).

\section{System Setup}

We simulated with the open-source software Optilux ${ }^{8}$ the transmission of a 19 -channel $112 \mathrm{~Gb} / \mathrm{s}$ PDM-QPSK homogeneous WDM system with 50 $\mathrm{GHz}$ channel spacing. All channels were first modulated by nested Mach-Zehnder modulators with independent 1024 De Bruijn sequences, and then their states of polarization (SOP) were randomized on the Poincaré sphere. Before creating the WDM comb, each channel was filtered over a $0.4 \mathrm{~nm}$ bandwidth. The simulated link was composed of $20 \times 100 \mathrm{~km}$ spans of single mode fiber (SMF), with zero overall cumulated dispersion obtained with a linear post-compensating fiber. Two different setups were considered: 1) a DM link with pre-compensation of $-650[\mathrm{ps} / \mathrm{nm}]$ and 30 [ps/nm/span] of in-line residual dispersion, and 2) a noDM link without pre- and in-line compensation. PMD was emulated only in the DM link, since in absence of dispersion management the interaction between PMD and Kerr nonlinearity is known to be negligible ${ }^{3}$.

The XPM suppressor, when used, was implemented by a demultiplexer followed by a bank of delay lines and a multiplexer, as sketched in Fig. 1. Each channel in the suppressor was delayed by $D$ [ps] with respect to its left neighbor in wavelength.

Fiber propagation was obtained by solving the 
Manakov-PMD equation through the split step Fourier algorithm ${ }^{9}$. Fiber birefringence and PMD were emulated by using 50 different random waveplates per span. We assumed flat gain amplifiers with $6 \mathrm{~dB}$ noise figure at each span end, although the entire link noise was loaded as a unique white noise source before detection. Such an approach neglects nonlinear phase noise, which is here negligible ${ }^{3}$. Before detection we perfectly compensated optical linear impairments, i.e. GVD and PMD, which allows us to focus entirely on the extra penalty coming from the interplay of linear and nonlinear distortions along the link.

The central channel was detected with a standard DSP based coherent receiver including: mixing with an ideal local oscillator, low pass filtering over a bandwidth of $17 \mathrm{GHz}$, sampling, phaserecovery with the Viterbi algorithm using 7 taps, decision, and finally differential decoding 4 .

We measured the bit error rate (BER) through the Monte Carlo algorithm by counting 100 errors ${ }^{10}$, and then converting the estimated BER to Q-factor. To take into account the stochastic nature of PMD, each BER was averaged over 40 different runs with different random seeds. Each seed corresponded to a different random pattern, SOP, and fiber waveplates realization. For a fair comparison, we used the same random realizations when testing different setups.

\section{Results and Discussions}

We first measured the impact of the XPM suppressor on the performance of the NRZ, aRZ or iRZ-based system in a DM link without PMD. To this aim we measured the Q-factor for each pulse format by varying the delay $D$. For a fair comparison, in the NRZ and aRZ case we set the power to $-1 \mathrm{dBm}$ while for $\mathrm{iRZ}$ we used $2 \mathrm{dBm}$. With this choice all formats experience similar nonlinear effects ${ }^{2}$. Fig. 2(a) shows the Q-factor vs. delay $D$. The error bars indicate the $Q$-factor standard deviation. We note that the XPM suppressor is effective for all formats, with an increasing Qfactor for increasing $D$, so that the best option is to maximize the decorrelation among channels. A complete decorrelation is reached after a delay of roughly 10 symbols (357 ps).

We next studied the impact of PMD on the same three pulse formats in a DM link without suppressor. Here we set the power to $-2 \mathrm{dBm}$ for $\mathrm{NRZ}$ and $\mathrm{aRZ}$ and to $1 \mathrm{dBm}$ for iRZ. In Fig. 2(b) we show the $\mathrm{Q}$-factor vs. average DGD.
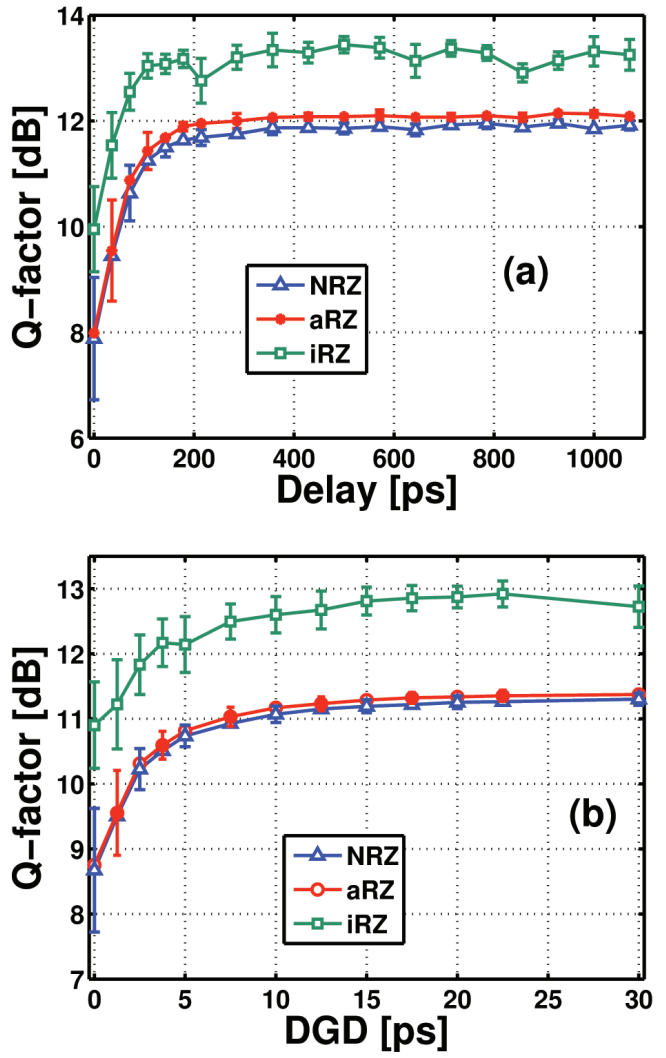

Fig. 2: $Q$-factor vs. suppressor delay at $\mathrm{DGD}=0$ (a) and vs. average DGD without suppressor (b) for the $20 \times 100$ $\mathrm{km}$ DM link with pulse formats NRZ, aRZ and iRZ.

This figure shows that the DGD improves the Qfactor for all pulse formats, and that it saturates for an average DGD larger than $20 \mathrm{ps}$, in agreement with $^{3}$.

We note that an average DGD of 5 ps improves $\mathrm{NRZ}$ and aRZ Q-factor by $2 \mathrm{~dB}$ compared with $D G D=0$, while for $i R Z$ the improvement is of only 1 $\mathrm{dB}$. The stochastic fluctuations of the Q-factor are mostly due to XPoIM and are related to the random, symbol-dependent SOP orientation of the PDM-QPSK signals. In fact, the standard deviation is larger at small DGD, where XPoIM is expected to be larger ${ }^{3}$. It is worth noting that $\mathrm{iRZ}$ has a smaller standard deviation than aRZ and $N R Z$ at $D G D=0$, since $i R Z$ is more tolerant to XPoIM in absence of PMD ${ }^{1}$. Note that the iRZ Q-factor increases for increasing DGD, even if PMD degrades the iRZ pulse-interleaving, because PMD-induced depolarization is more effective in reducing XPolM.

For the DM link and each pulse format, we also report in Fig. 3 the Q-factor vs. power in absence/presence of either PMD (average DGD $=0$ or $22.5 \mathrm{ps}$ ) or XPM-suppressor (delay $D$ equal to 0 or 10 symbols). As a reference, in the same 

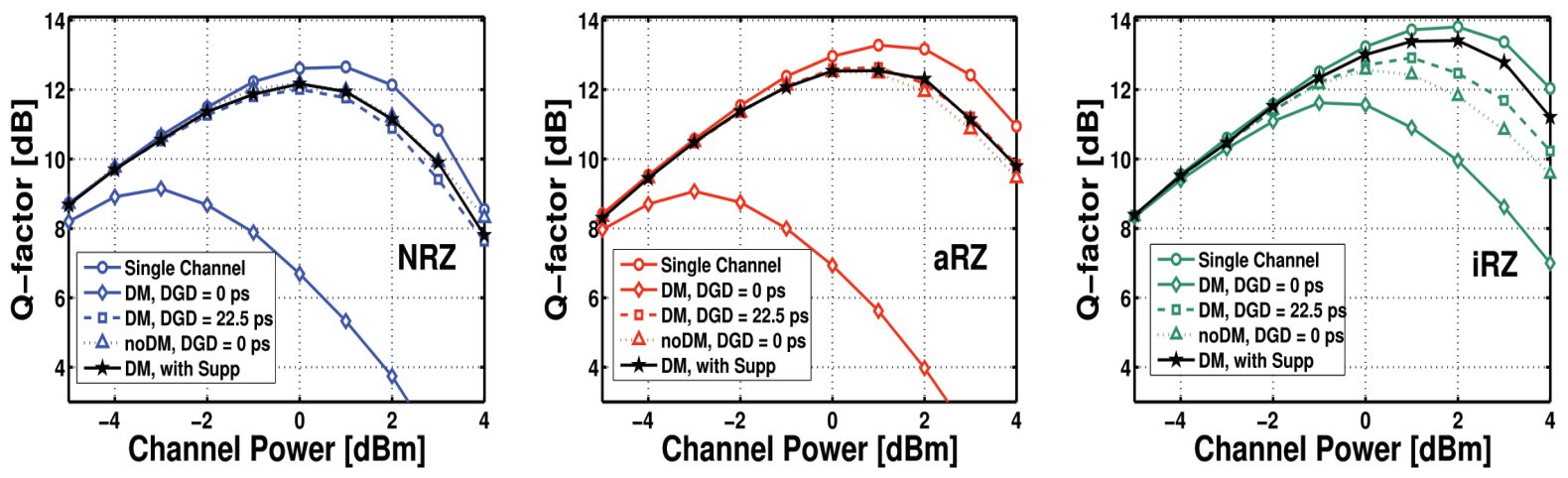

Fig. 3: Q-factor vs. power for different pulse formats and DGD.

graphs we also report the single channel DMcase and the WDM noDM-case, both in absence of DGD. The figure confirms that in absence of DGD the noDM link largely outperforms the DM one. However, PMD improves the DM performance yielding $Q$-factors very close to the noDM case, and even better with iRZ pulses. Again, we note for iRZ that the PMD-induced depolarization compensates for the degraded time-interleaving. From the figures, we also note that for aRZ and NRZ the DM link with XPM suppressor has similar performance as the noDM link, while for iRZ the DM link with XPM suppressor is superior to the noDM link, with a $Q$-factor very close to the singlechannel case. Reason is that the XPM suppressor reduces cross-channel interactions, but does not degrade pulse time-interleaving. It is thus the best option for a PDM-QPSK link with iRZ pulses.

In a final test we investigated the performance of the DM link with XPM suppressor in presence or absence of PMD. The Q-factor vs. power is reported in Fig. 4 for all pulse formats. We used the same average DGD and suppressor delay of Fig. 3. From Fig. 4 we note that PMD improves performance except for iRZ, where we observe a small decrease of the $Q$-factor in the nonlinear regime (descending region of $Q$-factor) making iRZ performance similar to aRZ. We ascribe such a worsening to the PMD-induced deterioration of the pulses' time-interleaving.

\section{Conclusions}

We investigated different solutions to mitigate cross-channel nonlinearities in $112 \mathrm{~Gb} / \mathrm{s}$ PDMQPSK transmissions. We showed that decorrelating the channels, through either PMD, or delay-line XPM suppressor, or by removing dispersion management, improves performance and reduces the gap among $i R Z, N R Z$, and aRZ pulse

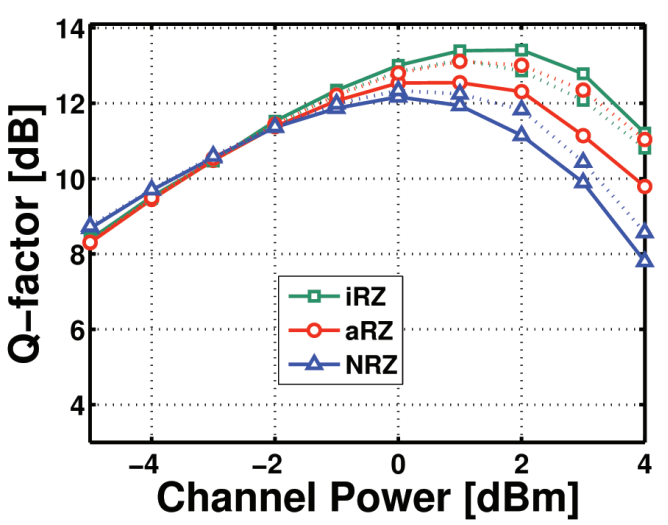

Fig. 4: Q-factor vs. power for a DM link with XPM suppressor (10-symbol delay) with average $D G D=0$ (solid line) and 22.5 ps (dotted line).

formats. We also showed that in iRZ-PDM-QPSK the worsening of the pulses' time-interleaving due to PMD is more than offset by the positive PMDinduced depolarization effect that reduces XPoIM. We find that the best option is to use iRZ-PDMQPSK in a DM link with an XPM suppressor at each span to decorrelate channels without neither compromising the time-orthogonality of the PDM tributaries, as with PMD, nor inducing more nonlinear self-effects, as with noDM.

\section{References}

1 C. Xie, IEEE Photon. Tech. Lett. 21, 274 (2009).

2 O. Bertran-Pardo et al., Opt. Express, 17, 19902 (2009).

3 P. Serena et al., Proc. ECOC'09, Th10.4.3 (2009).

4 G. Charlet et al., Proc. OFC08, OThU8 (2008).

5 G. Bellotti et al., Photon. Tech. Lett.12, 726 (2000).

6 C. Xie, Proc. ECOC'09, P4.08 (2009).

7 M. Winter et al., J. Lightw. Tech., 27, 3739 (2009).

8 P. Serena et al., "Optilux Toolbox", available at www.optilux.sourceforge.net.

9 C.R. Menyuk et al, J. Lightw. Tech. 24, 2806(2006).

10 J.C. Antona et al., Proc. ECOC '08, We1.E.3 (2008). 